\title{
Epidemiology of seasonal influenza infection in pregnant women and its impact on birth outcomes
}

\author{
A. K. REGAN ${ }^{1,2 *}$, H. C. MOORE ${ }^{3}$, S. G. SULLIVAN ${ }^{4,5,6}$, N. DE KLERK ${ }^{3}$ AND \\ P. V. EFFLER ${ }^{2}$ \\ ${ }^{1}$ School of Public Health, Curtin University, Bentley, WA 6152, Australia \\ ${ }^{2}$ Department of Health Western Australia, Communicable Disease Control Directorate, Perth, WA 6008, \\ Australia \\ ${ }^{3}$ Telethon Kids Institute, University of Western Australia, Subiaco, WA 6008, Australia \\ ${ }^{4}$ WHO Collaborating Centre for Influenza Research and Reference, Melbourne, VIC 3000, Australia \\ ${ }^{5}$ Melbourne School of Population and Global Health, University of Melbourne, Carlton, VIC 3053, Australia \\ ${ }^{6}$ Fielding School of Public Health, University of California, Los Angeles, CA 90095, USA
}

Received 4 March 2017; Final revision 27 July 2017; Accepted 9 August 2017; first published online 11 September 2017

\section{SUMMARY}

Seasonal influenza can cause significant morbidity in pregnant women. Much of the existing epidemiological evidence on influenza during pregnancy has focused on the 2009 A/H1N1 pandemic. To measure the epidemiological characteristics of seasonal influenza infection among pregnant women and the impact on infant health, a cohort of 86779 pregnancies during the influenza season (2012-2014) was established using probabilistic linkage of notifiable infectious disease, hospital admission, and birth information. A total of 192 laboratory-confirmed influenza infections were identified ( $2 \cdot 2$ per 1000 pregnancies), $14 \cdot 6 \%$ of which were admitted to hospital. There was no difference in the proportion of infections admitted to hospital by trimester or subtype of infection. Influenza B infections were more likely to occur in second trimester compared with influenza A/H3N2 and influenza A/H1N1 infections $(41 \cdot 3 \%, 23 \cdot 6 \%$, and $33 \cdot 3 \%$, respectively), and on average, infants born to women with influenza B during pregnancy had $4 \cdot 0 \%(95 \%$ CI $0 \cdot 3-7 \cdot 6 \%)$ lower birth weight relative to optimal compared with infants born to uninfected women $(P=0 \cdot 03)$. Results from this linked population-based study suggest that there are differences in maternal infection by virus type and subtype and support the provision of seasonal influenza vaccine to pregnant women.

Key words: Epidemiology, infant health, influenza A virus, influenza B virus, pregnant women.

\section{INTRODUCTION}

Seasonal influenza causes an estimated 3-5 million cases of severe disease and 250-500 000 deaths globally each year [1]. In Australia, influenza is implicated in at

\footnotetext{
* Author for correspondence: Dr A. K. Regan, School of Public Health, Curtin University, GPO Box U1987, Perth, WA 6845, Australia.

(Email: Annette.Regan@curtin.edu.au)
}

least 1400 deaths [2], 18000 hospitalizations, and over 300000 general practice consultations [3] each year. Pregnant women have an increased risk of hospital admission with influenza compared with non-pregnant women [4-6]. Physiologic and immunologic changes which occur during pregnancy can influence a woman's ability to respond to infection, resulting in serious disease [7].

Epidemiological studies have documented the burden of pandemic influenza among pregnant women 
and its impact on perinatal health $[8,9]$. However, fewer studies have examined seasonal influenza infection among pregnant women, and to our knowledge, no study has measured maternal infection by seasonal influenza virus type or subtype. Furthermore, although it is generally accepted that antenatal influenza infection increases the risk of adverse birth outcomes [10], the evidence documenting the association between seasonal influenza on infant health is mixed [11].

To investigate the epidemiology of seasonal influenza infection during pregnancy, we used population-based data linkage as a method for identifying laboratoryconfirmed influenza infections and associated hospital admissions and birth outcomes with the intention of: (a) comparing seasonal influenza during pregnancy by trimester and influenza virus subtype; and (b) evaluating the impact of seasonal influenza infections on perinatal health.

\section{METHODS}

We conducted a retrospective cohort study using a data linkage of notified influenza cases among pregnant women. We aggregated routine administrative health data from four population-based data sources to identify women who gave birth, their medical history (including influenza infection), and their birth outcomes.

\section{Pregnancy information}

Pregnancy status was determined based on the Midwives Notification System, a state-mandated collection of perinatal data. This data source includes information on $>99 \%$ of births in Western Australia based on information provided by the medical professional attending the birth (most frequently a midwife) [12]. Information provided included the woman's age, weight, height, and pre-existing chronic medical conditions, including diabetes and asthma. The woman's prepregnancy height and weight were used to calculate body mass index (BMI) to measure obesity (defined as a $\mathrm{BMI} \geqslant 30$ ).

\section{Birth outcomes}

The Midwives Notification System also collects information on birth, including the date of delivery, estimated gestation, and infant weight. The system also provides calculated estimates of proportion of optimal birth weight, proportion of optimal body length, and proportion of optimal head circumference. The system uses maternal height, age, parity, and infant sex as predictors of individual infant's optimal birth weight, head circumference, and body weight based on fractional polynomial regression models $[13,14]$. The proportion of optimal birth weight, body length, and head circumference is then estimated based on the ratio of the anthropometric measurement to the projected optimal measurement. These measures have been previously validated [14] and are likely to be a more reliable method of measuring extreme values of relative growth restriction. These measures were included as continuous measures of intrauterine growth.

\section{Laboratory-confirmed influenza infection}

Laboratory-confirmed influenza is a notifiable condition in Australia and in the state of Western Australia; these data are stored in the Western Australia Notifiable Infectious Diseases Database. Laboratory confirmation of influenza infection can be determined by either virus culture, nucleic acid testing, virus antigen testing, or seroconversion [15]. More than $88 \%$ of laboratory confirmation is made by reverse transcriptase polymerase chain reaction (RT-PCR). Notification data include virus type and subtype, diagnostic method, date of onset, and date of specimen collection.

\section{Hospital separation data}

Hospital separations were identified based on the state's hospital discharge data, known as the Hospital Morbidity Data Collection [16]. The database includes information provided in discharge summaries from all public and private hospitals in the state. Data include principal and additional diagnoses (International Classification of Diseases, 10th edition (ICD-10)-Australia Modification (AM) code), date of admission, date of discharge, and days spent in an intensive care unit.

\section{Data linkage and analysis}

All deliveries of a live or stillborn infant with gestation $\geqslant 20$ weeks between 15 March 2012 and 31 December 2014 (most recently available) according to the Midwives Notification System were linked with influenza notifications and hospital separations. Individuals' information was probabilistically matched by the Data Linkage Branch at the Department of Health Western Australia based on identifying information about the woman. 
Date of conception was calculated based on the baby's date of birth and estimated gestation. A woman's pregnancy period was defined as the time between the estimated date of conception through to the date of birth. A woman was considered to have seasonal influenza infection during pregnancy if she had a record of laboratory-confirmed influenza with a date of specimen collection during her pregnancy period. Influenza hospital admissions were defined as any admission to hospital with an admission date within 7 days of the date of specimen collection. Women with a pregnancy period coinciding entirely outside the influenza season were excluded from analyses.

Data analyses were conducted in SAS version 9.4 (SAS Institute, Cary, North Carolina, USA). Analyses were restricted to influenza cases occurring within influenza seasonal periods. The influenza season was defined based on the number of notifications received by the Department of Health Western Australia between 2012 and 2014. The start and end of the season were determined based on seasonal threshold values (see Supplementary Fig. S1).

We compared influenza infection by maternal characteristics, trimester of pregnancy, and influenza virus type and subtype. Log-binomial regression models were used to estimate the relative risk (RR) of hospitalization among laboratory-confirmed infections by maternal characteristics and influenza characteristics. For women with complete information on birth outcomes, we compared outcomes of pregnancies with laboratory-confirmed influenza infection to outcomes in pregnancies with no record of influenza infection $(\alpha=0 \cdot 05)$. Comparisons of categorical variables were made using Cochran-Mantel-Haenszel tests; continuous variable comparisons were made using independent $t$-tests or Mann-Whitney $U$ tests, depending on the distribution of the variable.

\section{Ethics}

Ethics approval was provided by the Department of Health Western Australia Human Research Ethics Committee (RA\#2013/71), the Western Australia Aboriginal Health Ethics Committee (RA\#536), and the University of Western Australia Human Research Ethics Committee (RA\#2016/6095).

\section{RESULTS}

During the 2012-2014 influenza seasons, 95801 pregnancies were identified; 8994 women who were not pregnant at any time during an influenza season were excluded from analyses (Supplementary Fig. S2). A total of 220 laboratory-confirmed influenza infections were identified, 192 of which occurred during the defined influenza season: 64 in 2012, 40 in 2013, and 88 in 2014. Most cases $(88 \cdot 2 \%)$ were confirmed by RT-PCR. Half the antenatal influenza cases $(50 \cdot 5 \%)$ were among women who conceived in the summer months (January through March; Fig. 1). The majority of cases were between 25 and 34 years of age $(60 \cdot 5 \%) ; 4.0 \%$ were Indigenous and $34.5 \%$ had a chronic medical condition; $14.0 \%$ self-reported smoking during pregnancy (Table 1).

Of the 192 laboratory-confirmed infections during influenza season, $48(25 \cdot 0 \%)$ infections were subtyped as $\mathrm{A}(\mathrm{H} 1 \mathrm{~N} 1)$ pdm09, $72(37 \cdot 5 \%)$ were $\mathrm{A} / \mathrm{H} 3 \mathrm{~N} 2,46$ $(24.0 \%)$ were influenza $\mathrm{B}$, and $26(13 \cdot 5 \%)$ were unsubtyped influenza A (Fig. 1). The relative frequency of each virus type/subtype varied by year, and some gestational differences were observed by influenza virus type and subtype. While $64.6 \%$ of infections with A (H1N1)pdm09 and $66.7 \%$ of infections with A (H3N2) occurred during the third trimester, $43 \cdot 5 \%$ of infections with influenza $\mathrm{B}$ occurred during the third trimester $\left(\chi^{2}=6 \cdot 31 ; P=\cdot 01\right) ; 15 \cdot 2 \%$ of influenza $\mathrm{B}$ infections occurred during the first trimester; and $41 \cdot 3 \%$ occurred during the second trimester (Fig. 2).

\section{Influenza hospitalization}

Twenty-eight $(14.6 \%)$ women with a notified, laboratory-confirmed infection were hospitalized. The mean length of stay in hospital was 1.7 days (s. D. $1 \cdot 0)$. Nearly half of admitted women $(46 \cdot 4 \%)$ had a principal diagnosis of 'diseases complicating pregnancy and the puerperium' (ICD-10-AM codes O98-99); $35 \cdot 7 \%$ were diagnosed with influenza (ICD-10-AM codes J10 and J11) or asthma (ICD-10-AM code J45); 17.9\% had a principal diagnosis consistent with a pregnancy complication (ICD-10-AM code O21-44). All 28 admissions were non-delivery admissions to hospital.

Among influenza-positive pregnant women, those with asthma were more than twice as likely to be hospitalized with influenza compared with women with no known pre-existing medical condition (RR 2.61 (95\% CI 1.13-6.04)) and had a significantly longer length of stay in hospital (2.5 and 1.6 days, respectively, $P=0 \cdot 03$ ). By trimester of pregnancy, the proportion of women infected with influenza who were 


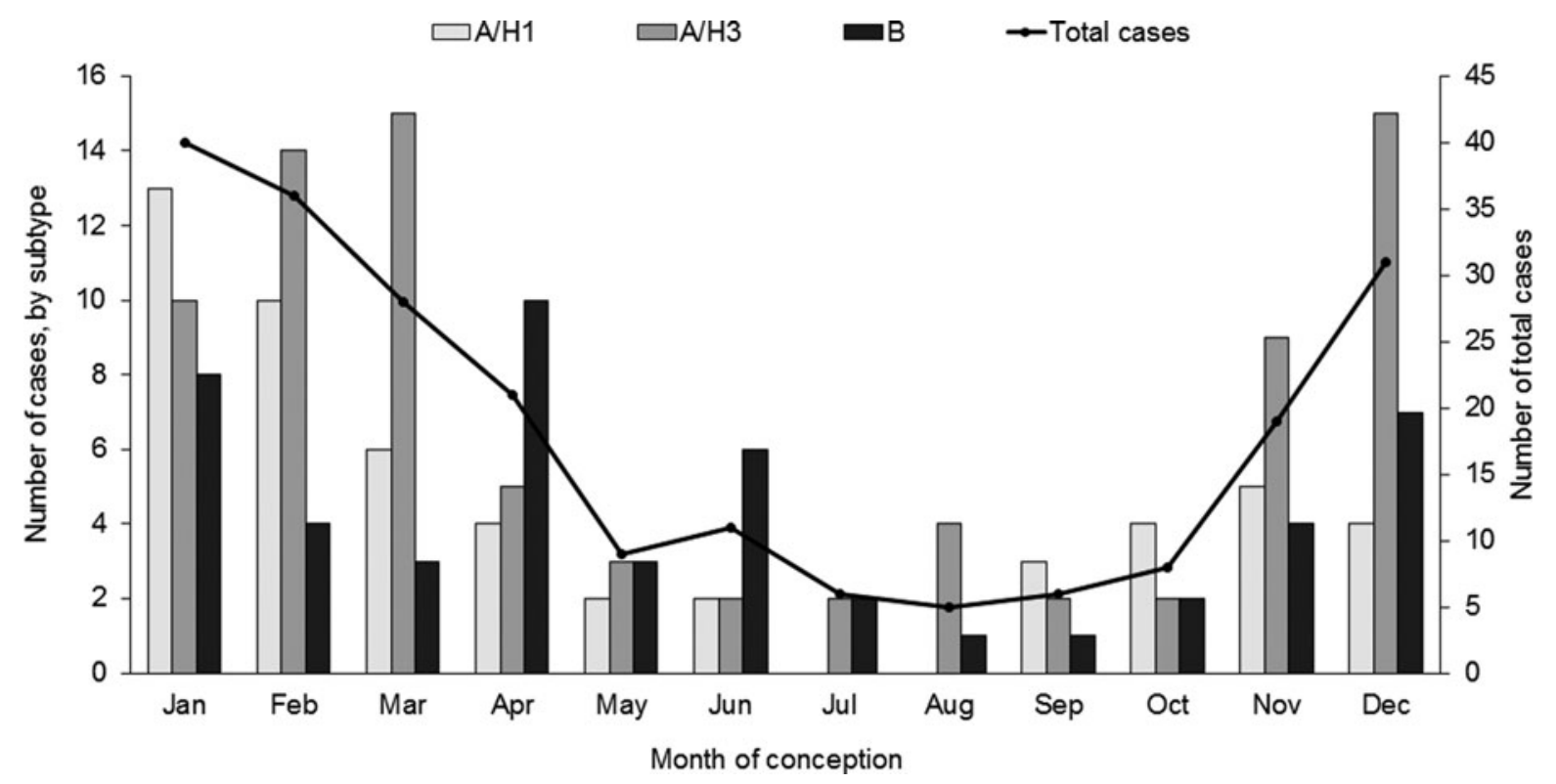

Fig. 1. Seasonal influenza infections in pregnant women, by month of conception and virus type and subtype $(n=220)-$ Western Australia, 2012-2014.

admitted to hospital was $17 \cdot 7 \%$ in their first trimester, $20.3 \%$ in their second trimester, and $11.2 \%$ in their third trimester. There was no difference in the percent of cases hospitalized by trimester of infection $(P=$ $0 \cdot 17)$ or by subtype of infection $(P=0 \cdot 21)$.

\section{Birth outcomes}

A total of 191 pregnant women with laboratoryconfirmed influenza and 86052 pregnant women with no record of laboratory-confirmed influenza during their pregnancy had complete information on birth outcomes. Birth outcomes among women who were infected with influenza during pregnancy were generally similar to those of women with no record of influenza (Fig. 3). On average, infants born to women infected with influenza during pregnancy were a healthy weight (mean $=3348.8 \mathrm{~g}, 95 \%$ CI $3267 \cdot 0-3430 \cdot 5 \mathrm{~g}$ ), full-term deliveries (median $=39$ weeks, IQR 38-40 weeks), and were near optimal birth weight $($ mean $=98 \cdot 7 \%, 95 \%$ CI $96 \cdot 8-100 \cdot 5 \%$ ). Similarly, infants born to women with a laboratoryconfirmed influenza requiring hospitalization were a healthy weight $($ mean $=3368.2 \mathrm{~g}, 95 \%$ CI $3079 \cdot 3-$ $3647 \cdot 2 \mathrm{~g}$ ), full-term deliveries (median $=39$ weeks, IQR 38-39 weeks), and were near their optimal birth weight $($ mean $=101 \cdot 6 \%, 95 \%$ CI $96 \cdot 7-106 \cdot 5 \%$ ).

However, some differences by trimester of infection and virus type and subtype were observed. For example, women infected during the first trimester had significantly lower median gestation (median $=$ 38 weeks, IQR 38-39 weeks) compared with women with no record of influenza $(P=0 \cdot 01)$. Infants born to women infected in second trimester had a $0.9 \%$ (95\% CI $0 \cdot 1-1 \cdot 6 \%$ ) lower mean measurement of head circumference relative to optimal compared with infants born to women with no record of influenza $(P=0 \cdot 03)$. No other statistically significant differences by trimester were observed. Infants born to women infected with influenza B during pregnancy had a $4 \cdot 0 \%(95 \%$ CI $0 \cdot 3-7 \cdot 6 \%)$ lower mean percent of optimal body weight compared with infants born to uninfected women $(P=0.03)$ (Fig. 4). Other measurements of fetal growth, birth weight, and gestation did not differ significantly, and no such differences were observed for influenza A/H1N1 or influenza A/H3N2.

\section{DISCUSSION}

This study highlights the potential use of linked population-level data to measure the health impacts of influenza in pregnant women, a target population for annual vaccination. Based on these data, we identified 192 laboratory-confirmed influenza infections in Western Australia during the 2012, 2013, and 2014 southern hemisphere influenza seasons, reflecting an incidence of $2 \cdot 2$ influenza cases per 1000 pregnancies. Fifteen percent of pregnant women with influenza were admitted to hospital. We observed no difference 
Table 1. Seasonal influenza infection during pregnancy and percent of cases admitted to hospital-Western Australia, 2012-2014

\begin{tabular}{|c|c|c|c|c|c|c|}
\hline & \multirow{2}{*}{$\begin{array}{l}\text { Total births } \\
2012-2014 \\
n(\%)\end{array}$} & \multicolumn{2}{|c|}{ Laboratory-confirmed influenza } & \multicolumn{2}{|c|}{$\begin{array}{l}\text { Percent of laboratory-confirmed } \\
\text { influenza cases admitted to } \\
\text { hospital }\end{array}$} & \multirow{2}{*}{$\begin{array}{l}\text { Length of stay in hospital for } \\
\text { influenza (in days) } \\
\text { Mean (s.D.) range }\end{array}$} \\
\hline & & No. of cases & Cases per 1000 women & $n(\%)$ & $\operatorname{RR}(95 \% \mathrm{CI})^{\mathrm{a}}$ & \\
\hline Total & $86779(100)$ & 192 & $2 \cdot 2$ & $28(14 \cdot 6)$ & - & $1 \cdot 7(1 \cdot 0) 1-4$ \\
\hline \multicolumn{7}{|l|}{ Birth year } \\
\hline 2012 & $20144(23 \cdot 2)$ & 64 & $3 \cdot 2$ & $17(26 \cdot 6)$ & Ref & $1 \cdot 7(0 \cdot 9) 1-3$ \\
\hline 2013 & $32544(37 \cdot 5)$ & 40 & $1 \cdot 2$ & $8(20 \cdot 0)$ & $0 \cdot 75(0 \cdot 36-1 \cdot 58)$ & $1 \cdot 9(1 \cdot 0) 1-4$ \\
\hline 2014 & $34091(39 \cdot 3)$ & 88 & $2 \cdot 6$ & ISD & ISD & ISD \\
\hline \multicolumn{7}{|l|}{ Maternal age (in years) } \\
\hline$<25$ & $15353(17 \cdot 7)$ & 32 & $2 \cdot 1$ & $7(21 \cdot 9)$ & Ref & $1 \cdot 3(0 \cdot 7) 1-3$ \\
\hline $25-29$ & $24769(28 \cdot 5)$ & 60 & $2 \cdot 4$ & $12(20 \cdot 0)$ & $0 \cdot 91(0 \cdot 40-2 \cdot 09)$ & $1 \cdot 8(0 \cdot 9) 1-3$ \\
\hline $30-34$ & $28747(33 \cdot 1)$ & 57 & $2 \cdot 0$ & $5(8 \cdot 8)$ & $0 \cdot 40(0 \cdot 14-1 \cdot 16)$ & $1 \cdot 4(0 \cdot 5) 1-2$ \\
\hline$\geqslant 35$ & $17910(20 \cdot 6)$ & 43 & $2 \cdot 4$ & ISD & ISD & ISD \\
\hline \multicolumn{7}{|l|}{ Indigenous status } \\
\hline Indigenous & $4944(5 \cdot 7)$ & 8 & $1 \cdot 6$ & ISD & ISD & ISD \\
\hline Non-Indigenous & $81835(94 \cdot 3)$ & 184 & $2 \cdot 2$ & $24(13 \cdot 0)$ & - & $1 \cdot 7(1 \cdot 0) 1-4$ \\
\hline \multicolumn{7}{|l|}{ Region of residence $^{\mathrm{b}}$} \\
\hline Metropolitan & $67991(78 \cdot 5)$ & 144 & $2 \cdot 1$ & $20(13 \cdot 9)$ & $0.83(0.39-1 \cdot 76)^{\mathrm{c}}$ & $1 \cdot 8(1 \cdot 0) 1-4$ \\
\hline Northern non-metropolitan & $6327(7 \cdot 3)$ & 25 & $3 \cdot 9$ & ISD & ISD & ISD \\
\hline Southern non-metropolitan & $12299(14 \cdot 2)$ & 23 & $1 \cdot 9$ & ISD & ISD & ISD \\
\hline \multicolumn{7}{|l|}{ Socio-economic status ${ }^{\mathrm{d}}$} \\
\hline Lowest $20 \%$ & $15219(18 \cdot 4)$ & 45 & $2 \cdot 9$ & $10(22 \cdot 2)$ & Ref & $1 \cdot 8(1 \cdot 0) 1-3$ \\
\hline $20-39 \%$ & $17183(20 \cdot 8)$ & 37 & $2 \cdot 1$ & $8(21 \cdot 6)$ & $0 \cdot 97(0 \cdot 43-2 \cdot 21)$ & $1 \cdot 6(0 \cdot 9) 1-3$ \\
\hline $40-59 \%$ & $16838(20 \cdot 4)$ & 27 & $1 \cdot 6$ & ISD & ISD & ISD \\
\hline $60-79 \%$ & $19101(23 \cdot 1)$ & 40 & $2 \cdot 1$ & ISD & ISD & ISD \\
\hline Highest $20 \%$ & $14372(17 \cdot 4)$ & 39 & $2 \cdot 7$ & ISD & ISD & ISD \\
\hline \multicolumn{7}{|l|}{ Self-reported smoking } \\
\hline Smoker & $9356(10 \cdot 8)$ & 28 & $3 \cdot 0$ & $7(25 \cdot 0)$ & $1 \cdot 95(0 \cdot 92-4 \cdot 16)$ & $2 \cdot 3(0 \cdot 9) 1-3$ \\
\hline Non-smoker & $77423(89 \cdot 2)$ & 164 & $2 \cdot 1$ & $21(12 \cdot 8)$ & Ref & $1.6(0 \cdot 9) 1-4$ \\
\hline \multicolumn{7}{|l|}{ Chronic medical conditions } \\
\hline No condition identified & $65717(75 \cdot 7)$ & 128 & $1 \cdot 9$ & $14(10 \cdot 9)$ & Ref & $1 \cdot 6(0 \cdot 9) 1-3$ \\
\hline Any condition ${ }^{\mathrm{e}}$ & $21062(24 \cdot 3)$ & 64 & $3 \cdot 0$ & $14(21 \cdot 9)$ & $2 \cdot 00(1.02-3.94)^{\mathrm{f}}$ & $1 \cdot 9(1 \cdot 1) 1-4$ \\
\hline Asthma & $4915(5 \cdot 7)$ & 21 & $4 \cdot 3$ & $6(28 \cdot 6)$ & $2 \cdot 61(1 \cdot 13-6 \cdot 04)^{\mathrm{f}}$ & $2 \cdot 5(1 \cdot 2) 1-4^{\mathrm{g}}$ \\
\hline Obesity & $17059(19 \cdot 7)$ & 49 & $2 \cdot 9$ & $7(14 \cdot 3)$ & $1 \cdot 31(0 \cdot 56-3 \cdot 04)$ & $1 \cdot 3(0 \cdot 5) 1-2$ \\
\hline Diabetes & $313(0 \cdot 4)$ & $<5$ & ISD & ISD & ISD & ISD \\
\hline
\end{tabular}




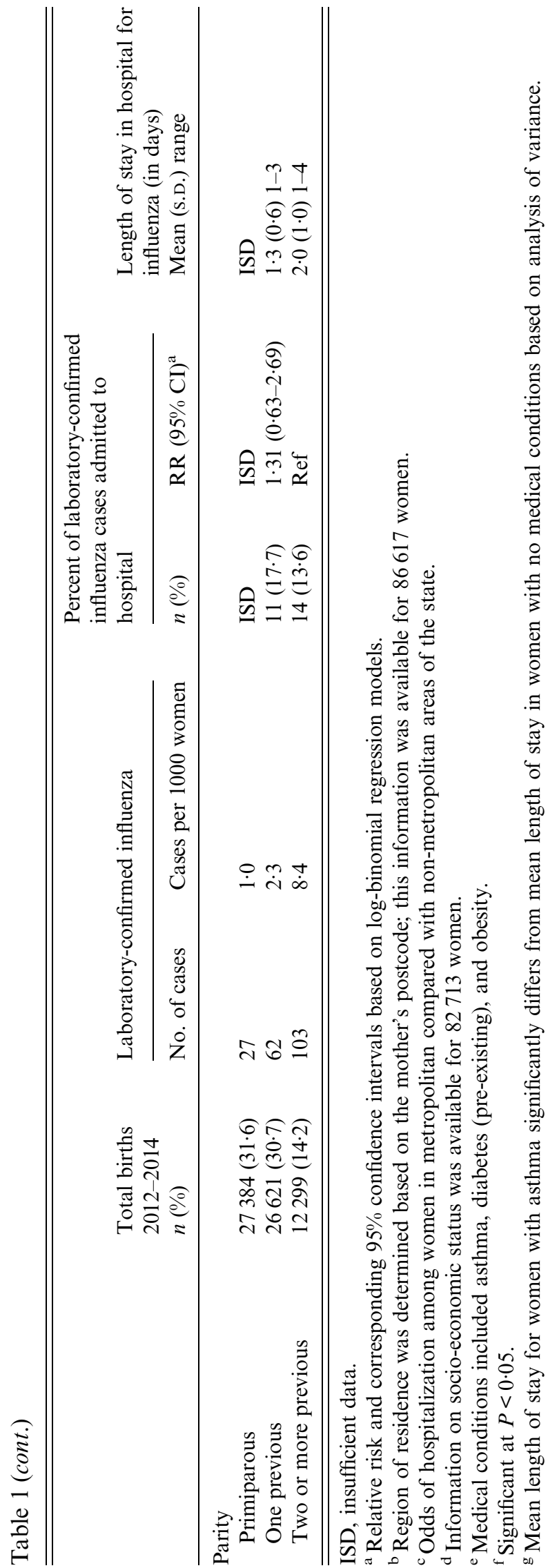

in the likelihood of admission to hospital in first, second, or third trimester infections.

The proportion of pregnant women infected with influenza and admitted to hospital has not been well described in the literature. The majority of studies have been restricted to hospital-based samples, and do not include community cases [17]. Our findings suggest that one in six pregnant women with laboratoryconfirmed influenza is hospitalized. However, several factors may have influenced these results. First, it is possible that testing is more common in a hospital setting, which would have artificially inflated these estimates. However, pathology testing records from our state suggest that testing of pregnant women hospitalized with an acute respiratory illness is not routine and only around $11 \%$ are tested for influenza (author's unpublished data). Second, by comparison with nonpregnant adults, pregnant women are more likely to be admitted for observation or based on obstetric concerns, which does not necessarily reflect increased severity of influenza disease [17]. Additional research from prospective cohorts could provide further information on the severity of seasonal influenza infection during pregnancy.

Few studies have been able to measure outcomes at birth following seasonal influenza infection in pregnancy. A recent systematic review showed that research on preterm birth and fetal death following antenatal influenza infection is limited [11]. Among the existing studies, researchers observed a significant association between antenatal infection with pandemic H1N1 and adverse birth outcomes [11], although some studies have failed to observe any impact on birth outcomes [18]. We observed some differences in gestation and growth by trimester of infection. Women infected with seasonal influenza during their first trimester had slightly lower mean duration of gestation compared with uninfected women. Previous studies of severe antenatal infections with either seasonal or pandemic influenza have documented an increased risk of preterm birth [9, 19] and small-for-gestational age births $[8,20]$, and these conditions can have persistent health impacts [21].

With the exception of A(H1N1)pdm09 infections, we are not aware of previous studies that have explored pregnancy and birth outcomes by virus type and subtype. In our study, a greater proportion of influenza B infections occurred earlier in pregnancy compared with influenza A infections. Moreover, infants born to women infected with influenza B had lower measurements of body weight relative to optimal growth 


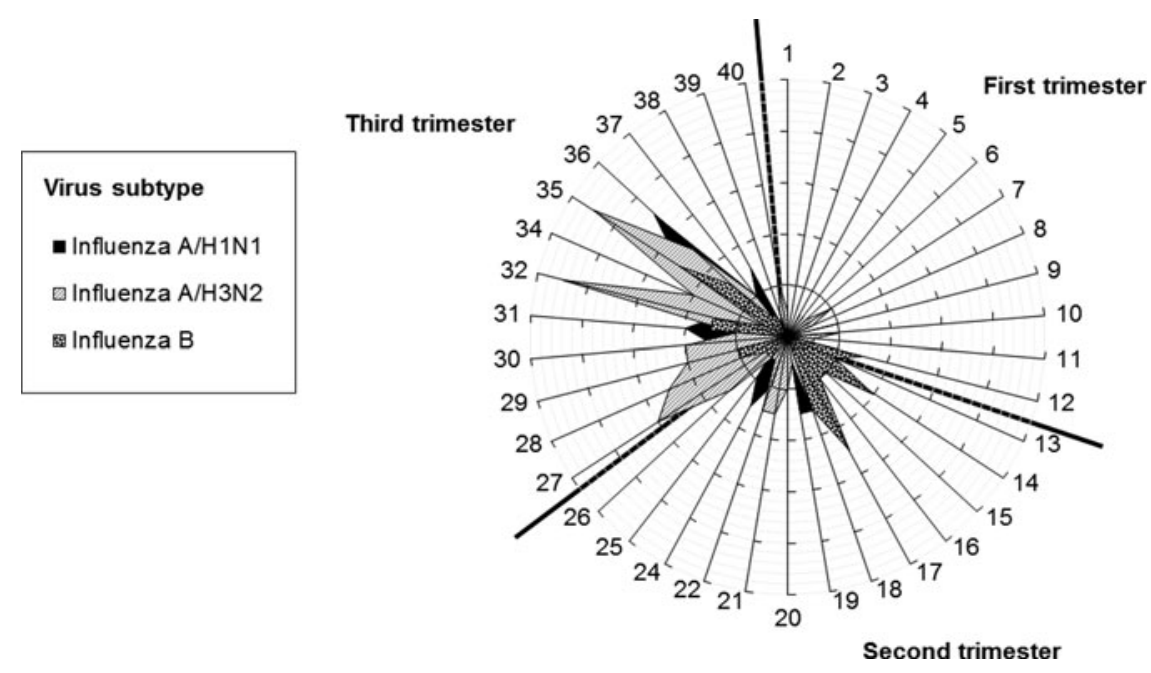

Fig. 2. Distribution of seasonal influenza infections in pregnant women, by week of gestation $(n=192)-$ Western Australia, 2012-2014.
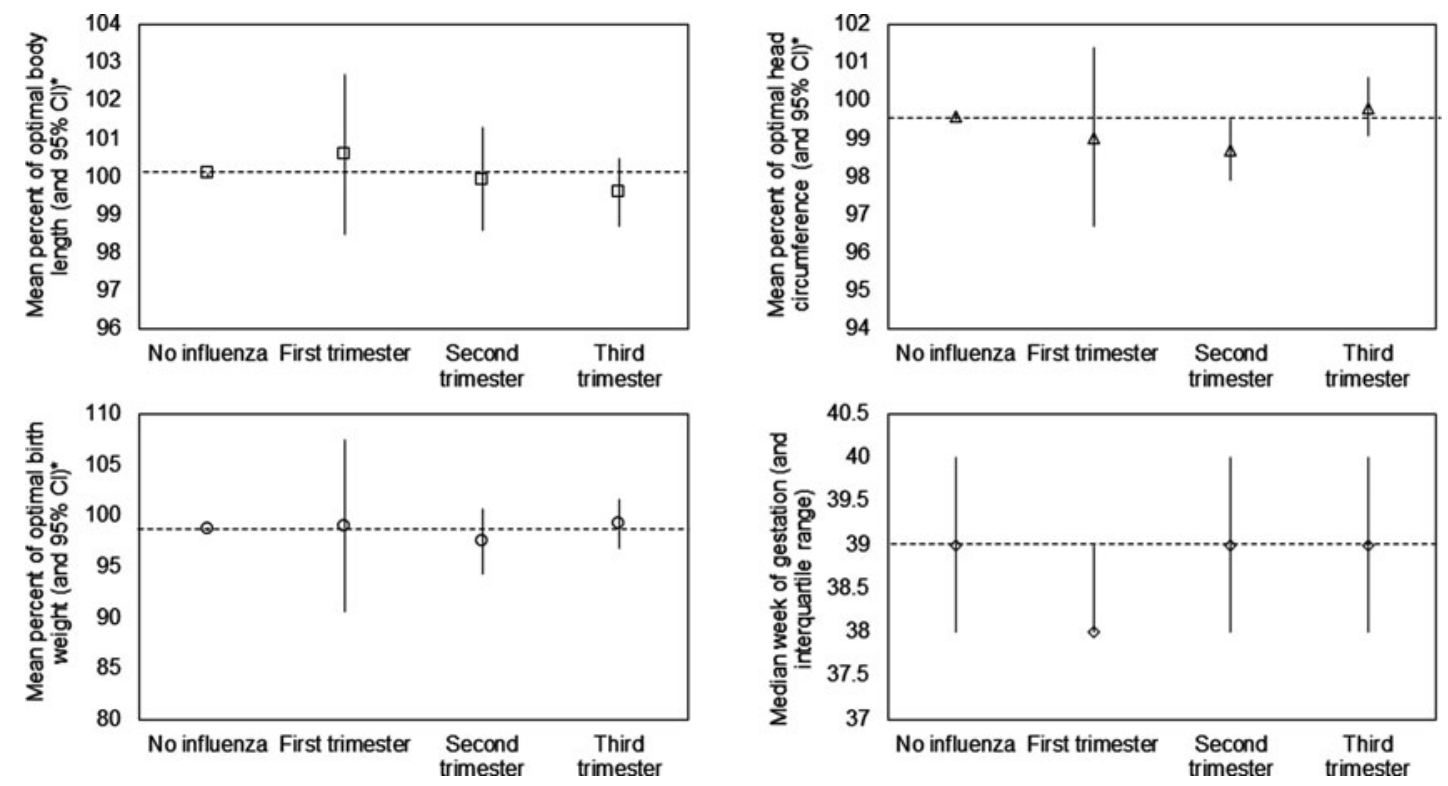

Fig. 3. Mean percent of optimal body length, head circumference and body weight, gestation, and infant weight among women infected with influenza during pregnancy $(n=191)$ and births in the general population $(n=86052)$, by trimester of infection - Western Australia, 2012-2014.

compared with infants born to uninfected women. During the seasons studied, influenza B circulation was evident (largely B/Yamagata strains), but it was never the dominant virus. Influenza $\mathrm{A}(\mathrm{H} 3 \mathrm{~N} 2)$ dominated in 2012 and 2013 and influenza $\mathrm{A}(\mathrm{H} 1 \mathrm{~N} 1)$ pdm09 dominated in 2014 [22-24]. Because influenza $\mathrm{B}$ is rarely the dominant virus, far less emphasis has been placed on its role in disease burden [25, 26]. However, evidence is mounting that influenza B infections may contribute a greater burden than previously recognized. For example, influenza B has been reported to be an important cause of severe illness in adults in the USA [27]. Among children, influenza B may cause significant morbidity [28] and elevated rates of infection among children could put pregnant mothers at risk. Other data from Western Australia suggest that, while laboratory-confirmed infections with influenza B are outnumbered by infections with influenza $A$, the risk of a severe outcome (hospitalization or death) from infection with $\mathrm{B} /$ Yamagata may be similar to $\mathrm{A}$ (H3N2) [29]. Our observations among pregnant women provide further support that the burden of 

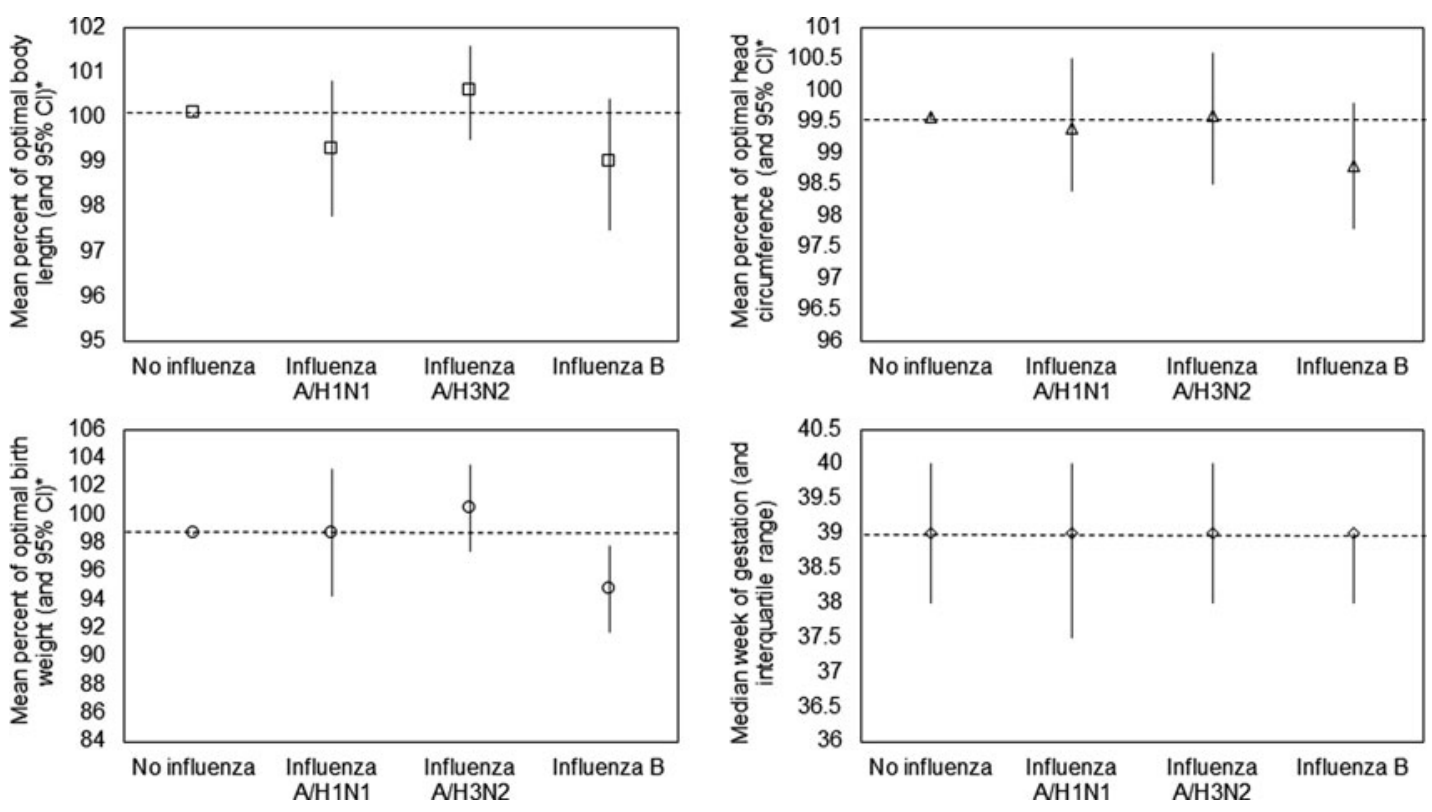

Fig. 4. Mean percent optimal body length, head circumference and birth weight, gestation, and infant weight among women infected with influenza $(n=191)$ and women with no record of influenza infection $(n=86052)$, by subtype of infection - Western Australia, 2012-2014.

influenza B may be underappreciated. In tropical regions, where influenza $\mathrm{B}$ infections may occur yearround [30], the role of influenza $B$ in adverse pregnancy outcomes may be more pronounced.

These results may have implications for vaccination policy. Current recommendations in Australia are to immunize pregnant women in any trimester of pregnancy [31]. This recommendation may be especially relevant to women with chronic medical conditions, particularly asthma, who we and others [32] have observed to have higher rates of influenza-associated hospitalizations. Although current recommendations state that influenza vaccination should be administered to pregnant women prior to the influenza season regardless of their trimester [31], studies on antenatal uptake of influenza vaccination in pregnancy have shown that vaccination early in pregnancy is uncommon [33]. The outcomes following first trimester influenza infection we observed suggest that there may be benefits to vaccination early in pregnancy. However, given the limitations of our sample and the limited amount of research documenting the benefits of influenza vaccination in the first trimester [34], further study is needed in this area.

Although we observed significantly shorter gestation and lower measures of relative birth weight by trimester and virus type, it is important to note that these differences may not necessarily translate into clinically meaningful endpoints, such as preterm delivery or low birth weight. The small number of pregnancies with laboratory-confirmed influenza reduced the precision of our estimates and made certain analyses impractical. Our sample was not adequately powered to compare traditional dichotomous measures of preterm birth, low birth weight, or small for gestational age. We were also unable to compare birth outcomes among women hospitalized with influenza by trimester of infection or to perform analyses of low incidence outcomes, such as fetal death. Given the limitations posed by our sample size, similar analyses with a larger sample of pregnancies with laboratory-confirmed infection, particularly in early pregnancy, would be informative for measuring the impact of antenatal influenza infection on perinatal health.

There are several additional limitations to this study. First, while the use of administrative datasets was an efficient method for creating a cohort of births with a large amount of health information, there is the possibility of measurement error in this study. The data collections linked in this study are considered to be high quality. The Midwives Notification System data are subject to rigorous review and the validity of variables often exceeds $98 \%$ [12]. Similarly, hospital inpatient data are thought to be well recorded, as in-house data quality activities and external audits of hospital medical records are regularly carried out [35]. Second, the cohort was defined based on birth records with gestation $\geqslant 20$ weeks 
gestation. As a result, earlier pregnancy loss could not be captured in our analyses. As previous studies suggest, there may be an increased risk of spontaneous abortion associated with antenatal influenza infection [36, 37], and additional studies which can capture early pregnancy loss would be beneficial. Further, because inclusion in the cohort was based on date of birth, we were unable to include women who were pregnant during the 2014 influenza season but did not deliver until after December 2014 in our dataset. As a result, women infected in early pregnancy in the 2014 season may be under-represented in our cohort. Finally, due to the nature of the datasets used, we were unable to measure maternal deaths. Maternal death is uncommon in Australia, with no more than six maternal deaths anticipated during the study period [38].

\section{CONCLUSION}

Seasonal influenza epidemics continue to place pregnant women at increased risk for severe complications [10]. Because of this risk (and potential benefits to young infants), pregnant women are the highest priority group for annual vaccination [39]. Results from this large population-based cohort study show that there may be epidemiological differences by influenza virus subtype and highlight the need for further investigation of the potential implications of antenatal influenza infection for infant health.

\section{SUPPLEMENTARY MATERIAL}

The supplementary material for this article can be found at https://doi.org/10.1017/S0950268817001972.

\section{ACKNOWLEDGEMENTS}

This study was funded by the Communicable Disease Control Directorate, Department of Health Western Australia. HCM was supported by an Early Career Fellowship of the National Health and Medical Research Council (APP1034254). The authors would like to thank the Linkage and Client Services Teams at the Data Linkage Branch (Department of Health Western Australia), in particular Alexandra Merchant and Mikhalina Dombrovskaya, as well as the Data Custodians Maureen Hutchinson (Midwives Notification System), Dr Gary Dowse (Western Australia Notifiable Infectious Disease Database), and Jessica George (Hospital Morbidity Data Collection).

\section{AUTHOR CONTRIBUTIONS}

AKR performed all data management and analyses and led the writing of the manuscript; HCM, SGS, $\mathrm{NdK}$, and PVE each contributed to the study design, interpretation of data, and writing of the manuscript. The findings were presented in brief during a latebreaking oral abstract session at the Options for Control of Influenza meeting in Chicago, Illinois in August 2016.

\section{DECLARATION OF INTEREST}

AKR, HCM, SGS, NdK, and PVE have no potential conflicts of interest to disclose.

\section{REFERENCES}

1. World Health Organization. Influenza (Seasonal) (http:// www.who.int/mediacentre/factsheets/fs211/en/). Accessed 18 January 2017.

2. Muscatello DJ, et al. Mortality attributable to seasonal and pandemic influenza, Australia, 2003 to 2009, using a novel time series smoothing approach. PLOS ONE 2013; 8: e64734.

3. Newall AT, Scuffham P, Hodgekinson B. Economic report into the cost of influenza to the Australian Health System (March 2007): Influenza Specialist Group 2007 (http://isg.org.au/assets/assets/isg-cost-influenza-report30-2007.pdf).

4. Dodds L, et al. Impact of influenza exposure on rates of hospital admissions and physician visits because of respiratory illness among pregnant women. Canadian Medical Association Journal 2007; 176: 463-468.

5. Meijer WJ, et al. Influenza virus infection in pregnancy: a review. Acta Obstetricia et Gynecologica Scandinavica 2015; 94: 797-819.

6. Neuzil KM, et al. Impact of influenza on acute cardiopulmonary hospitalizations in pregnant women. American Journal of Epidemiology 1998; 148: 1094-1102.

7. Gaunt G, Ramin K. Immunological tolerance of the human fetus. American Journal of Perinatology 2001; 18: 299-312.

8. Hansen C, et al. A large, population-based study of 2009 pandemic influenza A virus subtype H1N1 infection diagnosis during pregnancy and outcomes for mothers and neonates. Journal of Infectious Diseases 2012; 206: 1260-1268.

9. Pierce M, et al. Perinatal outcomes after maternal 2009/ H1N1 infection: national cohort study. British Medical Journal 2011; 342: d3214.

10. Rasmussen SA, Jamieson DJ, Uyeki TM. Effects of influenza on pregnant women and infants. American Journal of Obstetrics \& Gynecology 2012; 207: S3-S8.

11. Fell DB, et al. Maternal influenza and birth outcomes: systematic review of comparative studies. British Journal of Obstetrics and Gynecology 2016; 124: 48-59. 
12. Downey F. A Validation Study of the Western Australian Midwives' Notification System. 2005 Data. Perth: Department of Health Western Australia, 2007.

13. Blair EM, et al. Optimal fetal growth for the Caucasian singleton and assessment of appropriateness of fetal growth: an analysis of a total population perinatal database. BMC Pediatrics 2005; 5: 13.

14. Pereira G, Blair E, Lawrence D. Validation of a model for optimal birth weight: a prospective study using serial ultrasounds. BMC Pediatrics 2012; 12: 73.

15. Department of Health Western Australia. Influenza (http://www.public.health.wa.gov.au/3/1557/3/influenza. pm). Accessed 15 January 2017.

16. Department of Health Western Australia. Hospital Morbidity Data System (http://www.health.wa.gov.au/ healthdata/resources/hmds.cfm). Accessed 15 January 2017.

17. Mertz D, et al. Pregnancy as a risk factor for severe outcomes from influenza virus infection: a systematic review and meta-analysis of observational studies. Vaccine 2017; 35: 521-528.

18. Divala TH, et al. Incidence and seasonality of influenzalike illnesses among pregnant women in Blantyre, Malawi. American Journal of Tropical Medicine and Hygiene 2016; 95: 915-917.

19. Doyle TJ, Goodin K, Hamilton JJ. Maternal and neonatal outcomes among pregnant women with 2009 pandemic influenza A(H1N1) illness in Florida, 2009-2010: a population-based cohort study. PLOS ONE 2013; 8: e79040.

20. McNeil SA, et al. Effect of respiratory hospitalization during pregnancy on infant outcomes. American Journal of Obstetrics \& Gynecology 2011; 204: S54-S57.

21. Miller JE, et al. Association of gestational age and growth measures at birth with infection-related admissions to hospital throughout childhood: a populationbased, data-linkage study from Western Australia. Lancet Infectious Diseases 2016; 16: 952-961.

22. Australian Government Department of Health. Australian influenza report 2012-29 September to 12 October 2012 (\#10/2012) (https://health.gov.au/internet/main/publishing.nsf/Content/823D875A340A9A08CA257BF0001B1 D7D/\$File/ozflu-no8-2012.pdf). Accessed 21 December 2016.

23. Australian Government Department of Health. Australian influenza report 2013-28 September to 11 October 2013 (\#09/2013) (https://www.health.gov.au/internet/main/publishing.nsf/Content/FE0403E04324AAD0CA257BF0001 AFD91/\$File/australian-influenza-surveillance-report-022013.pdf). Accessed 21 December 2016.

24. Australian Government Department of Health. Australian Influenza Surveillance Report No 08-27 September to 10 October 2014 (http://www.health.gov. au/internet/main/publishing.nsf/Content/442C4236CCA 652BDCA257D58001736FE/\$File/Australian-InfluenzaSurveillance-Report.pdf). Accessed 21 December 2016.
25. Barr IG, Vijaykrishna D, Sullivan SG. Differential age susceptibility to influenza B/Victoria lineage viruses in the 2015 Australian influenza season. Eurosurveillance 2016; 21: pii=30118.

26. Vijaykrishna $\mathbf{D}$, et al. The contrasting phylodynamics of human influenza B viruses. eLife 2015; 4: e05055.

27. Su S, et al. Comparing clinical characteristics between hospitalized adults with laboratory-confirmed influenza $\mathrm{A}$ and $\mathrm{B}$ virus infection. Clinical Infectious Diseases 2014; 59: 252-255.

28. Harvala $\mathbf{H}$, et al. Burden of influenza B virus infections in Scotland in 2012/13 and epidemiological investigations between 2000 and 2012. Eurosurveillance 2014; 19: pii: 20903.

29. Giele C, et al. The differential burden of influenza B in Western Australia. Paper presented to Options IX for Control of Influenza, Chicago, IL, 24-28 August, 2016. http://2016.isirv.org/.

30. Saha S, Chadha M, Shu Y. Divergent seasonal patterns of influenza types A and B across latitude gradient in Tropical Asia. Influenza and Other Respiratory Viruses 2016; 10(3): 176-184.

31. Royal Australian and New Zealand College of Obstetricians and Gynaecologists. Influenza vaccination during pregnancy (C-Ob 45) (https://www.ranzcog.edu. au/RANZCOG_SITE/media/DOCMAN-ARCHIVE/ Influenza $\% 20$ vaccination $\% 20 \mathrm{in} \% 20$ pregnancy $\% 20(\mathrm{C}-\mathrm{Obs}$ $\% 2045) \% 20$ Review\%20Nov13.pdf). Accessed 2 February 2017.

32. Chaw L, et al. Burden of influenza and respiratory syncytial virus infection in pregnant women and infants under 6 months in Mongolia: a prospective cohort study. PLOS ONE 2016; 11: e0148421.

33. Centers for Disease Control and Prevention. Seasonal influenza vaccination coverage among women who delivered a live-born infant - 21 states and New York City, 2009-10 and 2010-11 influenza seasons. MMWR Morbidity \& Mortality Weekly Report 2013; 62: 1001-1004.

34. Skowronski DM, De Serres G. Is routine influenza immunization warranted in early pregnancy? Vaccine 2009; 27: 4754-4770.

35. Australian Institute of Health and Welfare. Australian hospital statistics 2012-13 (http://www.aihw.gov.au/ WorkArea/DownloadAsset.aspx?id=60129547000). Accessed 2 February 2017.

36. Giakoumelou $\mathbf{S}$, et al. The role of infection in miscarriage. Human Reproduction Update 2016; 22: 116-133.

37. Håberg SE, et al. Risk of fetal death after pandemic influenza virus infection or vaccination. New England Journal of Medicine 2013; 368: 333-340.

38. Australian Institute of Health and Welfare. Maternal deaths in Australia 2008-2012 (http://aihw.gov.au/ publication-detail/?id=60129551119). Accessed 2 February 2017.

39. World Health Organization. Vaccines against influenza WHO position paper - November 2012. Weekly Epidemiological Record 2012; 87: 461-476. 